\title{
Translation, cultural adaptation and validation for Brazilian Portuguese of the Cardiff Acne Disability Index instrument ${ }^{*}$
}

\author{
Luciana Rosa Grando ${ }^{1}$ \\ Vivian Trein Cunha ${ }^{1}$
}

\author{
Roberta Horn ${ }^{1}$ \\ Tania Ferreira Cestari ${ }^{1}$
}

DOI: http:/ / dx.doi.org/10.1590/abd1806-4841.20164402

\begin{abstract}
BACKGROUND:The Cardiff Acne Disability Index was originally developed in English for measuring quality of life of acne patients. Considering the psychosocial impact of this disease, it is important to have instruments culturally and linguistically validated for use in Brazilian adolescents.

Овлестіve: To translate the Cardiff Acne Disability Index into Brazilian Portuguese, culturally adapt it, and verify its reliability and validity in adolescent patients with acne.

Methods: In the first step, the Cardiff Acne Disability Index was translated and validated linguistically to Brazilian Portuguese in accordance with international guidelines published. In the second step, the validation of the Cardiff Acne Disability Index into Brazilian Portuguese instrument was performed, when patients aged from 12 to 20 years with acne were selected. The participants were interviewed to collect demographic data, submitted to the classification of acne by the Global Acne Grading System and invited to respond the Cardiff Acne Disability Index into Brazilian Portuguese version and DLQI ( $>16$ years) or CDLQI ( $\leq 16$ years). The internal consistency of Cardiff Acne Disability Index into Brazilian Portuguese was assessed by Cronbach's alpha coefficient and concurrent validity was measured by the Spearman correlation coefficient and Student 's t-test for paired samples. Results: The study included 100 adolescents. The Cardiff Acne Disability Index into Brazilian Portuguese version showed good reliability and internal consistency (Cronbach's alpha $=0.73$ ). The concurrent validity of the scale was supported by a strong and significant correlation with CDLQI / DLQI instruments ( $\mathrm{rs}=0.802 ; \mathrm{p}<0.001)$. Conclusions: Cardiff Acne Disability Index into Brazilian Portuguese version is a reliable, valid and valuable tool to measure the impact of acne on quality of life in adolescent patients.
\end{abstract}

Keywords: Acne vulgaris; Adolescent; Quality of life; Questionnaires; Translating; Validation studies

\section{INTRODUCTION}

Acne is a multifactorial disease of the pilosebaceous unit that has a peak incidence in adolescence, affecting up to $91 \%$ of boys and $79 \%$ of girls. ${ }^{1}$ The Global Alliance to Improve Outcomes in Acne recommended that acne is recognized as a chronic disease, while the social and economic effects related to it also make it a public health problem. ${ }^{2}$

More than an aesthetic nuisance, acne affects virtually every aspect of the individual's life: social, professional and academic. ${ }^{3}$ Studies have shown that acne can be associated with a spectrum of psychosocial abnormalities, including depression, suicidality, anxiety, psychosomatic symptoms, embarrassment and social inhibition. ${ }^{4-6}$ Further emphasizing how acne affects the life of the individual, levels of social, psychological and emotional problems in acne patients have been compared with the levels of patients suffering from debilitating chronic diseases such as asthma, epilepsy, diabetes, back pain and arthritis. ${ }^{7}$

There is no consensus in the literature that the clinical severity of acne and its impact on quality of life show a linear relation, given the fact that this dysfunction is dependent on the patient's capacity of adjustment when facing this condition. ${ }^{8}$ Assessing the impact of acne on quality of life requires specific, validated and reliable instruments that are brief and easy to administer and interpret. ${ }^{9}$

\section{Received on 25.01.2015}

Approved by the Advisory Board and accepted for publication on 18.05.2015 Study performed at Hospital de Clínicas de Porto Alegre - Universidade Federal de Ciências da Saúde de Porto Alegre (HCPA-UFRGS) and in private clinics
- Porto Alegre (RS), Brazil.

Financial support: None.

Conflict of interest: None.

Universidade Federal de Ciências da Saúde de Porto Alegre (UFRGS) - Porto Alegre (RS), Brazil.

(C2016 by Anais Brasileiros de Dermatologia 
Motley and Finlay were the first authors to publish questionnaires to assess the quality of life of patients with acne. Initially they formulated the ADI (Acne Disability Index), a scale consisting of 10 items that is strongly correlated to the severity of acne. ${ }^{3}$ From the ADI, CADI (Cardiff Acne Disability Index) was derived, a compound questionnaire with only 5 items, which proved to be a practical tool for dermatologists use in routine care. ${ }^{10}$

Recently, Brazilian authors published the AcneQoL-Br, a translated version of the Acne-QoL, culturally adapted and validated for the Portuguese spoken in Brazil. ${ }^{11,12}$ The instrument consists of 19 questions organized into four areas relating to facial acne: own perception, social aspect, emotional aspect and acne symptoms. The final questionnaire showed adequate reliability and validity for studies on facial acne.

Researchers on the theme recognize that among the specific scale validated to measure the patient's quality of life with acne, the ones that show better correlation with the severity of the disease and with the improvement after treatment are Acne-QoL and CADI, respectively. Due to the fact that it is a scale with fewer items, CADI was suggested as the best tool to use in clinical practice and Acne-QoL was proposed as the most appropriate in the context of clinical trials. ${ }^{13}$

CADI is a specific questionnaire that measures disability induced acne, especially designed for adolescents. It consists of 5 questions relating to the preceding month: Questions 1 and 2 address the psychological and social consequences of acne in general; Question 3 is directed to patients with acne in the trunk; Question 4 addresses the psychological state of the patient; and Question 5 asks the patient a subjective assessment of the acne severity. Each question has 4 possible responses, with a maximum of 3 points and a minimum of 0 and a maximum total score of 15 points. Scores from 0 to 5 denote a slight impact on quality of life; from 6 to 10, a moderate impact; and from 11 to 15 denotes a severe impairment. ${ }^{10}$

Originally developed in English, by its convenient use and high sensitivity, it has been translated into several languages, including French, Persian, Ukrainian, Chinese, Serbian and Arabic. ${ }^{13-20}$

The objective of this study is to translate and validate the instrument Cardiff Acne Disability Index (CADI) for the Portuguese spoken in Brazil - Questionnaire to assess quality of life in acne (CADI-PB).

\section{MATERIAL AND METHODS}

This was a cross-sectional study conducted in two sequential steps described below:

Phase I - Translation and cultural adaptation:

The process of translation and cultural adaptation of CADI questionnaire was conducted according to international standards and recommendations of authors for translation of quality of life instruments (QOL) in order to maintain equivalence between the original and the destination versions. ${ }^{21,22}$

The process' steps were: preparation (obtaining permission from the author to the questionnaire translation), translation (performed by two translators), reconciliation (review of differences of language and grammatical construction by a bilingual group, composed of professionals involved in the health area), pilot testing (applied in 10 acne patients), synthesis and reformulation, back-translation, review and finalization. The review committee of the translations and back-translations consisted of two bilingual dermatologists, a medical student and two certified translators.

\section{Phase II - Validation}

This cross-sectional study was conducted in the outpatient clinic of the Dermatology Department of the Hospital de Clínicas de Porto Alegre, at the Universidade Federal do Rio Grande do Sul, Brazil, and also in private clinics in the city of Porto Alegre-RS. The project was approved by the Ethics Committee of the University, which is consistent with the standards of the Declaration of Helsinki (2008) and Good Practices in Clinical Research (No: 8545712300005327). Participants (or their guardians) signed the informed consent prior to enrollment. Patients were selected as they sought the clinic voluntarily or were referred for treatment of facial acne. The study was conducted from July 2013 to July 2014.

The study included patients with active acne (predominance of comedones, papules, pustules and nodules), aged 12-20 years, and who agreed to participate in the study. Patients with predominance of acne scars and those who did not understand the Brazilian Portuguese were excluded from the study.

Participants and/or their guardians, after signing the informed consent (IC), were interviewed to obtain demographic data and were evaluated by trained examiners to determine the degree of acne through the Global Acne Grading System (GAGs). ${ }^{23}$ This system considers 6 locations, including 5 regions in the face and 1 in the trunk. For each region, one FACTOR is assigned. For each type of acneic lesions is given a value (GRADE), depending on the severity $(0=$ no lesion; 1 = comedones; 2 = papules; 3 = pustules; and $4=$ nodules). The score for each area is calculated by the formula: local score $=$ FACTOR $\times$ GRADE (0-4). The GLOBAL SCORE is the sum of local scores, which will determine the severity of acne: $1-18$ score is considered mild; $19-30$, moderate; $31-38$, severe; and $>39$, very severe. This system has proven to be very accurate and with minimal variability intra- and inter-observer, be- 
ing widely used in research involving acne grading. ${ }^{24}$

Then, patients were asked to answer 2 questionnaires. The first was the translated Portuguese version of CADI (CADI-PB). The second was the Quality of Life in Dermatology Index (DLQI) for patients $>16$ years or the Quality of Life Index in Children's Dermatology (CDLQI) for those $\leq 16$ years, both previously translated and validated for Portuguese spoken in Brazil. ${ }^{25,26}$

The reliability of an instrument is the degree that it is free from random error, that is, its ability to generate the same results when applied in the same group of subjects. ${ }^{27}$ To test this attribute we used:

- Internal consistency analysis for the questionnaire as a whole and for each domain, assessed by Cronbach $\alpha$ coefficient;

- Test-retest reproducibility, where we compared the responses of 2 successive administrations of the questionnaire in a group of 20 participants, with an interval of 14 days, using the intraclass correlation coefficient and the Student $\mathrm{t}$-test for paired samples.

The validity of an instrument is the degree in which it measures what it proposes to measure. ${ }^{27}$ To test this feature, we contemplated:

- Content validity though researchers committee analysis;

- Concurrent validity, in which CADI-PB answers were compared with previously validated instruments (DLQI and CDLQI) through the Spearman correlation coefficient;

- Construct validity, based on exploratory factor analysis.

All statistical analysis of data was performed using SPSS (Statistical Package for Social Sciences, version 13.0 for Windows, SPSS Inc., Chicago, IL).

\section{RESULTS}

Phase I - Translation and cultural adaptation:

The process of translation and cultural adaptation can be seen in the diagram of figure 1 . The final version of CADI-PB is shown in chart 1.

\section{Phase II - Validation:}

Mean age of study participants was $15.4 \pm 2.1$ years. Gender distribution was similar (56\% male and $44 \%$ female). Most patients (83\%) was white. Mean duration of acne varied widely among patients (mean and SD of $30.5 \pm 2.1$ months, respectively).

Acne lesions involve the face and trunk in 63\% of patients and only $37 \%$ in the face. When asked about family history of acne, $51 \%$ of patients confirmed it, $38 \%$ denied it and $11 \%$ did not know about acne history of their parents. Of the patients who reported a family history of acne, $24(47.1 \%)$ related it to the father, 20
(39.2\%) to the mother and $7(13.7 \%)$ to both.

Fifty percent of patients had already undergone treatment for acne, and of these, only 30\% (15 adolescents) were satisfied with the results and the majority (94\%) reported appearance of new acne lesions after treatment completion. The most cited modalities were isolated topical treatment (60\%) and combined with oral antibiotics (26\%). The demographic characteristics of the sample are shown in table 1.

In the evaluation of acne severity index, 58\% presented mild acne; $36 \%$, moderate; and $6 \%$ had severe acne. Mean CADI-PB score was $5.2( \pm 2.6)$ with a minimum of 0 and a maximum of 12 . The internal consistency assessed by Cronbach's alpha was 0.73, indicating good consistency of the instrument.

In the evaluation of test-retest reliability, questionnaires of the first and second evaluation showed a intra-class correlation coefficient (ICC) of 0.89 , demonstrating a good correlation between the two assess-

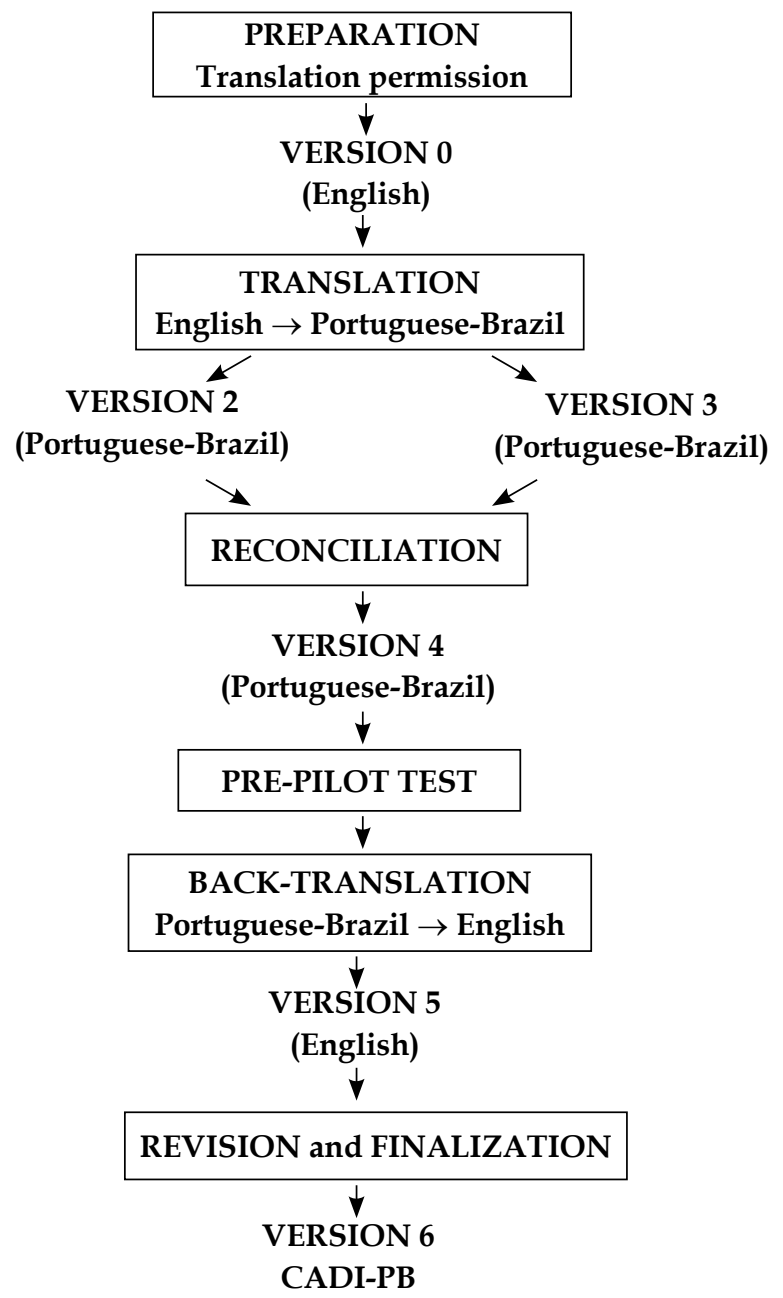

FIGURE 1: Diagram of the process of translation and cultural adaptation of CADI 
CHART 1: Final version of CADI-PB questionnaire

\section{CADI QUESTIONNAIRE}

Name:

Date:

Mark with $\mathrm{X}$ the best answer to each question:

1. Did your acne problem make you feel aggressive, frustrated or embarrassed in the last month?
$\square$ 3. Very much
$\square$ 2. Much
1. A little
0 . Not at all

2. Do you think having acne interferes with your daily social life, participation in social events or in relations with the opposite sex in the last month?

$\square$ 3. Severely, affecting all activities

$\square$ 4. Moderately, in most activities

5 . Occasionally or in only some activities

0. Not at all

3. During the last month, did you avoid attending

changing rooms or use bathing suit because of your

acne?
$\square$ 3.All the time
2. Most of times
4.Occasionally

Please check if you answered all the questions.

(c) Cardiff Acne Disability Index. R J Motley, A Y Finlay 1992

ments. In the comparison of the difference between the mean of the two evaluations through the Student t-test for paired samples there was no statistically significant difference $(\mathrm{t}=1.29 ; \mathrm{p}=0.212)$.

To test the construct validity, an exploratory factor analysis was used adopting the criterion of principal component analysis with Varimax rotation. This analysis showed that there are two factors explaining the scale. Factor 1 is composed of items that assess acne in general and explains $46.34 \%$ of the variance. Factor 2 includes the item related to trunk acne and explains $21.99 \%$ of the variance in the total sample. Together, Factors 1 and 2 explained $68.33 \%$ of the sample variability. Table 2 describes the rotated factor loads of CADI items.

The concurrent validity of CADI-PB version showed a significant association with the results of CDLQI/DLQI $\left(r_{s}=0.802 ; p<0.001\right)$. In graph 1 is shown the concurrent validity of the instruments (Graph 1).

There was a significant positive association between acne severity score and CADI total score, but with low intensity $\left(r_{s}=0.234 ; p=0.019\right)$.

\section{DISCUSSION}

Several studies have been conducted to evaluate the quality of life of patients with acne, using generic or specific instruments. Generic instruments are useful
4. How would you describe your feelings about the appearance of your skin in the last month?
3. Very depressed and unhappy
2. Generally worried
1. Occasionally worried
0. Unworried

5. Please indicate how you consider the severity of your acne now:
3. Never been so bad
1. A minor problem
2. A serious problem
0 . Not a problem

Thanks for your collaboration.

because they allow comparison of populations with different diseases, but they are limited by the lack of specificity regarding the disease and extent of questions, which can cause logistical difficulties in conducting a study. ${ }^{16}$ Researches confirm that specific instruments shown to be more responsive to changes after treatment, presumably because they measure variables that are really important for the patient with acne. ${ }^{28,29}$

Among some specific acne quality of life questionnaires, CADI was selected to be translated and validated for Portuguese spoken in Brazil by a certain reason. CADI is a self-administered quality of life scale developed specifically for young adults and adolescents. Having a small number of items (only 5), it can be answered quickly and therefore it is very convenient for use in the dermatologist's care routine. ${ }^{15}$

This study aims to develop a version for the Portuguese spoken in Brazil (CADI-PB) of CADI instrument, and therefore, it was in accordance with international recommendations for translation and validation of quality of life instruments (PRO - patient report outcome measures/ RRP - results reported by the patient instrument) (21) and met the requirements of reliability and reproducibility.

Mean CADI-PB score obtained in the study was 5.2, which indicates a slight impairment in the 
TABLE 1: Characterization of the sample of adolescent acne patients treated at the Hospital de Clínicas and at private clinics in the city of Porto Alegre-RS, Brazil

\begin{tabular}{|c|c|}
\hline Variables & $\mathbf{N}=100$ \\
\hline \multicolumn{2}{|l|}{ Age (years) } \\
\hline Median \pm SD & $15.4 \pm 2.1$ \\
\hline \multicolumn{2}{|l|}{ Gender $(\%)$} \\
\hline Male & 56 \\
\hline Female & 44 \\
\hline \multicolumn{2}{|l|}{ Race $(\%)$} \\
\hline White & 83 \\
\hline Black & 5 \\
\hline Brown & 12 \\
\hline \multicolumn{2}{|l|}{ Education (\%) } \\
\hline Incomplete elementary school & 36 \\
\hline Complete elementary school & 6 \\
\hline Incomplete high school & 39 \\
\hline Complete high school & 8 \\
\hline Higher education & 11 \\
\hline \multicolumn{2}{|l|}{ Acne duration (months) } \\
\hline Mean \pm SD & $30.5 \pm 15.4$ \\
\hline \multicolumn{2}{|l|}{ Sites affected (\%) } \\
\hline Face & 37 \\
\hline Face and trunk & 63 \\
\hline \multicolumn{2}{|l|}{ Family history (\%) } \\
\hline Yes & 51 \\
\hline No & 38 \\
\hline I don't know & 11 \\
\hline \multicolumn{2}{|l|}{ Treatment (\%) } \\
\hline Yes & 50 \\
\hline No & 50 \\
\hline \multicolumn{2}{|l|}{ Relapse after treatment (\%) } \\
\hline Yes & 94 \\
\hline No & 6 \\
\hline \multicolumn{2}{|l|}{ Acne severity (\%) } \\
\hline Mild & 58 \\
\hline Moderate & 36 \\
\hline Severe & 6 \\
\hline
\end{tabular}

TABLE 2: Rotated factor loads of the items of CADI-PB applied in the sample of adolescent acne patients treated at the Hospital das Clínicas and at private clinics in the city of Porto Alegre-RS, Brazil

\begin{tabular}{lll}
\hline CADI item & Factor $\mathbf{1}$ & Factor 2 \\
\hline Item 1 & 0.800 & 0.037 \\
Item 2 & 0.560 & 0.314 \\
Item 3 & 0.101 & 0.970 \\
Item 4 & 0.852 & 0.091 \\
Item 5 & 0.792 & 0.227 \\
\% of variance & 46.34 & 21.99 \\
\% cumulative & 46.34 & 68.33 \\
\hline
\end{tabular}

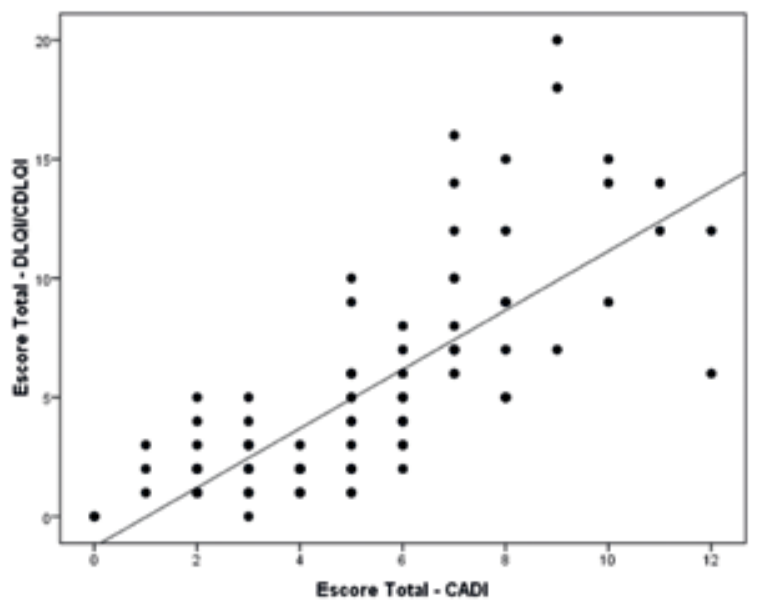

GraPH 1: Concurrent validation between CADI-PB and DLQI/ DLQI in the sample of adolescent acne patients assisted at the Hospital de Clínicas and private clinics in the city of Porto Alegre-RS, Brazil ( $\mathrm{rs}=0.802 ; \mathrm{p}<0.001$ )

quality of life of the population studied, according to most prior studies that used this method to evaluate the impact of acne on quality life..$^{8,19,24,30,31}$ Possibly this finding means the most commonly found proportion in cases of acne, where most patients present mild levels and the minority has more severe levels of the disease.

The proportions of acne cases by distribution by severity vary in the literature according to the division adopted (mild versus moderate and severe; mild versus moderate versus severe) as well as according to the origin of the patients (hospital versus outpatient). ${ }^{19}$ Recent studies using CADI reported prevalence rates of severe acne around $2.5 \%$ to $13.5 \%$, thus confirming that mild to moderate cases predominate and, in part, explaining the tendency of a mild impairment in the quality life according to the scores of this instrument. ${ }^{19,30}$

Although $50 \%$ of participants had been using treatments prior to the study, most of these $(70 \%)$ were unhappy with his acne problem. Possibly the therapeutic frustration experienced by these patients contributed to the negative impact on their quality of life.

Internal consistency, measured by Cronbach's alpha coefficient, was satisfactory $(0.73)$, certifying a good correlation between the questionnaire items. Previous studies that proposed to translate and validate CADI presented rates and Cronbach's alpha ranging from 0.76 to $0.87 . .^{15,31}$

There was no statistically significant difference between the first and second application of the questionnaire $(t=1.29 ; p=0.212)$ and the coefficient of intra-class correlation demonstrated strong correlation between the two evaluations (ICC $=0.89$ ), as it 
strengthens the reproducibility of the questionnaire and measurement stability.

The testing of the construct validity of CADI-PB has revealed the existence of two factors explaining the scale. Factor 1 covers general aspects of acne and explains $46.34 \%$ of the variance and Fator 2 includes the item related to trunk acne and explains $21.99 \%$ of the variance. Similar explanatory analyzes were performed in Serbian and Persian studies of translation and validation of CADI. Both identified two dimensions: emotional and social well-being. ${ }^{16,19}$

Researches show that when acne is concentrated on the face, the severity is related to general social aspects: embarrassment, shame, feelings of concern with the skin appearance and negative impacts on everyday social life. ${ }^{4,10,32}$ When located in the trunk, the severity of acne has been related to an impact on the self-awareness of the body and sexual appearance. Consistent with previous studies, these findings suggest a distinction between socially (face), sexually or emotionally (trunk) relevant areas of the body. ${ }^{33}$

In testing the concurrent validity of CADI-PB, a significant and satisfactory association with the results of CDLQI/DLQI questionnaires was evidenced $\left(\mathrm{r}_{\mathrm{s}}=\right.$ 0.802; $\mathrm{p}<0.001$ ), previously validated.

There was a significant positive association between acne severity score and CADI total score $\left(\mathrm{r}_{\mathrm{s}}=\right.$ $0.234 ; p=0.019)$, showing that the greater the severity of acne, the greater the impact on quality of life. However, we observed that the correlation has low intensity (Spearman's correlation coefficient $-r_{s}<0.3$ ). This finding is in agreement with the literature, where it is often observed that the correlation between the severity scores and impact on quality of life reported by the patient is of low intensity ${ }^{18,24,30,31}$ or even nonexistent. ${ }^{3,5,11,34}$

Although many measures are acceptable to assess the severity of acne, from simple and subjective clinical evaluation to more objective methods as the one used in this study, the patient's perception may include factors not identified by the dermatologist. Once the acne is highly visible and carries a certain degree of social negativity, it was hypothesized that even mild acne can lower self-confidence, influence on body image, and interfere with the desire to interact with others and to be seen in public. ${ }^{34}$

Adolescence, stage of development when we observe a peak incidence of acne, is another aspect that explains the disconnect between clinical severity and impact on quality of life. During this period the individual deals with fundamental issues such as changing in body image, sexuality, socialization, education, and vocational choices, among others. The patient's response to acne psychosocial effect can therefore vary considerably depending on the degree of security and confidence that the patient has in other areas of his development. $^{35}$

The realization that the quality of life scores in acne have greater correlation with the severity reported by patients than with the dermatologist assessment suggests that the perception of the disease by the patient is an useful account when assessing and conducting acne treatment. ${ }^{36}$

We identify some limitations of this study. Among them, the sampling method by convenience, not exclusively population-based, which could harm the generalization of the results. The fact that the study was performed in a sample from the Southern region on the country does not allow the extrapolation of the findings to other regions of Brazil, as it is a country marked by many differences in the Portuguese spoken, and in socio-educational and cultural matters. Also, in the selection of patients it was not contemplated an important exclusion criteria that could interfere with the results of this study. Patients with other chronic diseases and emotional disorders often suffer an impact on their quality of life, which could be a confounding factor in evaluating the results.

\section{CONCLUSION}

The results of this study show that the version for the Portuguese spoken in Brazil of CADI instrument (CADI-PB) is valid and reliable in addition to being a practical and useful tool in the assessment of patients with acne, adding relevant information to medical records under the adolescents' own perceptions.]

\section{ACKNOWLEDGEMENTS}

We thank professor Andrew Finlay (Department of Dermatology and Wound Healing, Medical School of Cardiff University - United Kingdom), for his formal permission to use CADI. We also thank Dr. Faraz Mahmood Ali (Clinical Research Fellow in Dermatology, Department of Dermatology and Wound Healing, Medical School of Cardiff University - United Kingdom) for the revision of back-translation and fast reply. 


\section{REFERENCES}

1. Tan JK, Vasey K, Fung KY. Beliefs and perceptions of patients with acne. J Am Acad Dermatol. 2001;44:439-45.

2. Gollnick HP, Finlay AY, Shear N; Global Alliance to Improve Outcomes in Acne. Can we define acne as a chronic disease? If so, how and when? Am J Clin Dermatol. 2008; 9: 279-84

3. Motley RJ, Finlay AY. How much disability is caused by acne? Am J Clin Dermatol. 2008;9:279-84

4. Kellett SC, Gawkrodger DJ. The psychological and emotional impact of acne and the effect of treatment with isotretinoin. Br J Dermatol. 1999:140:273-82.

5. Gupta MA, Gupta AK.. Depression and suicidal ideation in dermatology patients with acne, alopecia areata, atopic dermatitis and psoriasis. $\mathrm{Br} \mathrm{J}$ Dermatol. 1998;139:846-50.

6. Halvorsen JA, Stern RS, Dalgard F, Thoresen M, Bjertness E, Lien L. Suicida ideation, mental health problems, and social impairment are increased in adolescents with acne: a population-based study. J Invest Dermatol. 2011;131:363-70.

7. Mallon E, Newton JN, Klassen A, Stewart-Brown SL, Ryan TJ, Finlay AY. The quality of life in acne: a comparison with general medical conditions using generic questionnaires. Br J Dermatol. 1999;140:672-6.

8. Walker N, Lewis-Jones MS. Quality of life and acne in Scottish adolescent schoolchildren: use of the Children's Dermatology Life Quality Index. J Eur Acad Dermatol Venereol. 2006;20:45-50.

9. Rapp SR, Feldman SR, Graham G, Fleischer AB, Brenes G, Dailey M. The Acne Quality of Life Index (Acne-QOLI): development and validation of a brief instrument. Am J Clin Dermatol. 2006;7:185-92.

10. Motley RJ, Finlay AY. Practical use of a disability index in the routine management of acne. Clin Exp Dermatol. 1992;17:1-3.

11. Kamamoto Cde S, Hassun KM, Bagatin E, Tomimori J. Acne-specific quality of life questionnaire (Acne-QoL): translation, cultural adaptation and validation into Brazilian-Portuguese language. An Bras Dermatol. 2014;89:83-90.

12. Girman CJ, Hartmaier S, Thiboutot D, Johnson J, Barber B, DeMuro-Mercon C, et al. Evaluating health-related quality of life in patients with facial acne: development of a self-administered questionnaire for clinical trials. Qual Life Res. 1996;5:481-90.

13. Dreno, B. Assessing quality of life in patients with acne vulgaris: implications for treatment. Am J Clin Dermatol. 2006;7:99-106.

14. Dermatology.org.uk [Internet]. The Cardiff Acne Disability Index (CADI) [cited 2015 mar 28]. Available from: http://www.dermatology.org.uk/quality/cadi/ quality-cadi.html

15. Dreno B, Finlay AY, Nocera T, Verrière F, Taïeb C, Myon E. The Cardiff Acne Disability Index: cultural and linguistic validation in French. Dermatology. 2004;208:104-8.

16. Aghaei S, Mazharinia N, Jafari P, Abbasfard Z. The Persian version of the Cardiff Acne Disability Index: Reliability and validity study. Saudi Med J. 2006;27:80-2.

17. Chernyshov PV. Creation and validation of the Ukrainian version of the Cardiff Acne Disability Index. Lik Sprava. 2012;5:139-43.

18. Law MP, Chuh AA, Lee A. Validation of a Chinese version of the Cardifi Acne Disability Index. Hong Kong Med J. 2009;15:12-7.

19. Jankovic S, Vukicevic J, Djordjevic S, Jankovic J, Marinkovic J, Basra MK. The Cardiff Acne Disability Index (CADI): linguistic and cultural validation in Serbian. Qual Life Res. 2013;22:161-6.

20. Ismail KH, Mohammed-Ali KB. Quality of life in patients with acne in Erbil city. Health Qual Life Outcomes. 2012;10:60

21. Wild D, Grove A, Martin M, Eremenco S, McElroy S, Verjee-Lorenz A, et al. ISPOR Task Force for Translation and Cultural Adaptation. Principles of Good Practice for the Translation and Cultural Adaptation Process for Patient-Reported Outcomes (PR0) Measures: report of the ISPOR Task Force for Translation and Cultural Adaptation. Value Health. 2005;8:94-104.

22. Guillemin F, Bombardier C, Beaton D. Cross-cultural adaptation of healthrelated quality of life measures: literature review and proposed guidelines. J Clin Epidemiol. 1993;46:1417-32.

23. Doshi A, Zaheer A, Stiller MJ. A comparison of current acne grading systems and proposal of a novel system. Int J Dermatol. 1997;36:416-8.

24. Hanisah A, Omar K, Shah SA. Prevalence of acne and its impact on the quality of life in school-aged adolescents in Malaysia. J Prim Health Care. 2009;1:20-5.

25. Ferraz MB, Oliveira LM, Araujo PM, Atra E, Tugwell P. Crosscultural reliability of the physical ability dimension of the health assessment questionnaire. J Rheumatol. 1990;17:813-7.
26. Prati C, Comparin C, Boza JC, Cestari, TF. Validação para o português falado no Brasil do instrumento Escore da Qualidade de Vida na Dermatologia Infantil (CDLQI). Med Cutan Iber Lat Am. 2010;38:229-33.

27. Aaronson N, Alonso J, Burnam A, Lohr KN, Patrick DL, Perrin E, et al. Assessing health status and quality-of-life instruments: attributes and review criteria. Qual Life Res. 2002;11:193-205.

28. Klassen AF, Newton JN, Mallon E. Measuring quality of life in people referred for specialist care of acne: Comparing generic and disease-specific measures. J Am Acad Dermatol. 2000;43:229-33.

29. Hanna S, Sharma J, Klotz J. Acne vulgaris: more than skin deep. Dermatol Online J. 2003;9:8

30. Yap FB. Cardiff Acne Disability Index in Sarawak, Malaysia. Ann Dermatol. 2012;24:158-61.

31. Law MP, Chuh AA, Lee A, Molinari N. Acne prevalence and beyond: acne disability and its predictive factors among Chinese late adolescents in Hong Kong. Clin Exp Dermatol. 2010;35:16-21.

32. Papadopoulos L, Walker C, Aitken D, Bor R. The relationship between body location and psychological morbidity in individuals with acne vulgaris. Psycho Health Med 2000;5:431-8.

33. Hassan J, Grogan S, Clark-Carter D, Richards H, Yates VM. The individual health burden of acne: appearance-related distress in male and female adolescents and adults with back, chest and facial acne. J Health Psychol. 2009;14:1105-18.

34. Mulder MM, Sigurdsson V, van Zuuren EJ, Klaassen EJ, Faber JA, de Wit JB, et al. Psychosocial impact of acne vulgaris: Evaluation of the relation between a change in clinical acne severity and psychosocial state. Dermatology. 2001;203:124-30.

35. Uslu G, Sendur N, Uslu M, Savk E, Karaman G, Eskin M. Acne: prevalence, perceptions and effects on psychological health among adolescents in Aydin, Turkey. J Eur Acad Dermatol Venereol. 2008;22:462-9.

36. Martin AR, Lookingbill DP, Botek A, Light J, Thiboutot D, Girman CJ. Health-related quality of life among patients with facial acne - assessment of a new acnespecific questionnaire. Clin Exp Dermatol. 2001;26:380-5.
MAILING ADDRESS:

Luciana Rosa Grando

Avenida Luiza Manoel Gonzaga, 23/902

Petrópolis

90470-280 - Porto Alegre - RS

Brazil

Email: lucianarosa920@hotmail.com

How to cite this article: Grando LR, Horn R, Cunha VT, Cestari TF. Translation, Cultural Adaptation and Validation for Brazilian Portuguese of the Cardiff Acne Disability Index instrument . An Bras Dermatol. 2016;91(2):180-6. 